\title{
Poliomyelitis trends in Pondicherry, South India, 1989-91
}

\author{
D K Srinivasa, A Sahai, S B Rotti, M B Soudarssanane, M Danabalan, R N Sahoo, \\ K A Narayan, G Ramalingam, S Srinivasan, S Mahadevan, B V Bhat, P Nalini
}

\begin{abstract}
Study objectives-To assess the poliomyelitis trend, including study of the epidemiological features, and to correlate this with the immunisation coverage of infants. Design-Three annual lameness surveys in children aged 0-60 months employing cluster sampling methods and a series of five cross sectional surveys of immunisation coverage in children aged 12-23 months of age were undertaken. Setting-Pondicherry, India, 1988-92. Subjects-More than 10000 children in the age group of $0-60$ months took part in the three annual lameness surveys and samples of 210 children aged 12-23 months were covered each year in immunisation coverage surveys.
\end{abstract}

Measurements and main resultsAltogether 50 of 11461,24 of 10093 , and 17 of 11218 children surveyed during 1989 , 1990 , and 1991 respectively had become lame as a result of poliomyelitis, giving prevalences of $4.4,2.4$, and 1.5 per 1000 children for the three surveys. The corrected prevalences of poliomyelitis were $5.9,3.2$, and 2.0 per 1000 children during 1989,1990 , and 1991 respectively. The proportion of cases aged up to 36 months fell from $48 \%$ in 1989 to $12.5 \%$ in 1990 and $6 \%$ in 1991. The age at onset was less than 1 year in most. The median age at onset was 10.7 months. About $54 \%$ of the affected children had received three doses of oral poliomyelitis vaccine (OPV) before the onset of paralysis. In 1988 immunisation coverage for the third dose of OPV was $91 \%$ and in 1992 it was $97.6 \%$. The drop out rate for the first versus the third dose of OPV fell from $6.3 \%$ in 1988 to $1.9 \%$ in 1992.

Conclusion-Three successive annual lameness surveys showed that poliomyelitis was declining between 1989 and 1991. Five immunisation coverage surveys conducted from 1988 to 1992 showed high initial coverage followed by an improvement in the form of almost universal coverage for OPV.

( $($ Epidemiol Community Health 1997;51:443-448)

Jawaharlal Institute of Postgraduate Medical Education and Research,

Dhanvantari Nagar,

Pondicherry 605006

India

Correspondence to: Professor S B Rotti.

Accepted for publication January 1997
1985. Since then vaccination coverage has increased considerably.

Poliomyelitis is one of the major vaccinepreventable diseases. It was still rampant in many countries of the world between 1986 and 1988. The total numbers of cases reported to World Health Organization were 28951 and 35685 in 1986 and 1987 respectively and data reporting was incomplete for 1988 . Trends in regional incidence rates for poliomyelitis over a 15 year period from 1974-88 have suggested a decline, especially since $1982 .{ }^{1}$ In the national review of the Indian immunisation programme conducted in 1989, the prevalence of lameness due to poliomyelitis was $2.71 / 1000$ children in the 0-4 years age group. However, wide variation existed in different districts and urban zones. It was 0.39 in the northern district of Goa compared with the highest value of 8.77 in the district of Kanpur Dehat in Uttar Pradesh. ${ }^{2}$ Epidemics of poliomyelitis were reported in 1987 in Pondicherry and adjoining districts of Tamil Nadu, ${ }^{34}$ in southern Kerala, ${ }^{5}$ and in Delhi and its surrounding areas. ${ }^{6}$

In Pondicherry, since the vigorous implementation of the universal immunisation programme (UIP) in 1986, there has been all round progress in respect of manpower training and immunisation performance. This should have resulted in a reduction of disease occurrence, but regular disease surveillance may not be as efficient as it should be because many programmes are being carried out simultaneously. Because of this it was decided that yearly surveys of immunisation coverage and lameness surveys should be carried out in Pondicherry by an external agency (the Jawaharlal Institute of Postgraduate Medical Education and Research) which was not involved in the implementation of UIP. Immunisation coverage surveys were conducted for five years from 1988-92 and lameness surveys for three years from 1989-91. We report the coverage of immunisation and trends in the prevalence of poliomyelitis in Pondicherry together with some epidemiological features.

\section{Methods}

The Union Territory of Pondicherry has four administrative regions in different geographical areas, distributed over three states of India. These are Mahe in Kerala, Yanam in Andhra Pradesh, Karaikal and main Pondicherry close to Tamil Nadu. It had a population of 789416 in the 1991 census, scattered over an area of $492 \mathrm{~km}^{2}$. Vital rates obtained from sample survey system estimates for the year 1991 were 
very low. The crude birth rate was 18.9 and the crude death rate was 6.4 per thousand population. The infant mortality rate was very low compared with other Indian states. It was 33.9 per 1000 live births in 1991 , according to the report of the local health administration, ${ }^{7}$ and 27.1 per 1000 live births in 1987-88 in a cohort of 8185 in Pondicherry.

\section{ORGANISATION OF HEALTH SERVICES}

The health service in Pondicherry consists of 10 major hospitals including Jawaharial Institute of Postgraduate Medicine and Research, four community health centres, 18 primary health centres, 73 subcentres, and 30 dispensaries, including 11 under the "employees state insurance scheme". ${ }^{7}$ In addition to this, there were 677 anganwadis of the "integrated child development services scheme" (ICDS) supporting the immunisation and other health programmes.

\section{INTEGRATED CHILD DEVELOPMENT SERVICES SCHEME}

The ICDS has been in existence for 20 years. It is a multi sectoral programme involving several government departments, and their services are coordinated at the village, block, district, state, and central government levels. The department of women and child development has the primary responsibility of implementing the programme at the centre, whereas sub-branches in the states include social welfare, rural development, tribal welfare, and health and family welfare. The beneficiaries are children below 6 years, pregnant and lactating women, and women in the age group of 15 to 44 years.

The ICDS package of services includes supplementary nutrition, vitamin $\mathrm{A}$, iron and folic acid distribution, immunisation, and health check ups. An "anganwadi" is the focal point for the delivery of the services to children and mothers at their door steps. An anganwadi normally covers a population of 1000 in both rural and urban areas and 700 in tribal areas. Services at the anganwadi are delivered by an anganwadi worker, who is a part-time worker who receives an honorarium. She is assisted by a helper, who is also a local woman and is paid a small honorarium. The work of anganwadi workers is supervised by full time workers, the mukhya sevikas. They are appointed in the proportions of one per 25,20, and 17 anganwadis in urban, rural, and tribal projects resepectively. They are supervised by child development project officer. In the health department, medical officers of primary health centres, female health workers, and female health assistants are responsible for supervising the anganwadi workers. ${ }^{9}$

\section{UNIVERSAL IMMUNISATION PROGRAMME}

The UIP was launched in November 1985 in Pondicherry, which was divided into four districts for its implementation. One deputy director of immunisation and one cold chain officer at the state level and four district im-

\section{KEY POINTS}

- In Pondicherry, the prevalence of lameness due to poliomyelitis fell from 4.4 per 1000 children aged 5 or less in 1989 to 1.5 per 1000 in 1991.

- The universal immunisation programme was successfully implemented achieving 87$89 \%$ coverage for the third dose of oral polio vaccine with minimum drop-out rates.

- Fifty four per cent of lame children had received three doses of vaccine before the onset of paralysis.

- Apart from routine immunisation, annual vaccine campaigns on Child Protection Day (introduced since 1990) and pulse polio immunisation (since 1995-96) could further reduce or eradicate poliomyelitis in the region.

munisation officers at district level along with other support staff were responsible for implementation. Equipment was supplied in 1988-89 and 1989-90. This included 21 icelined refrigerators, 20 chest freezers, 34 cold boxes; 65 vaccine carriers, 28 carriers, and 41 dial thermometers. Vaccine potency tests were done regularly. All the five samples of vaccines sent in 1989-90 and 27 samples sent in 1990-91 were found to be satisfactory. All the medical officers, health assistants, health workers, village health guides, anganwadi workers, and trained birth attendants were given several courses of training under the UIP during 1986-89.

All the medical institutions file regular monthly reports on morbidity and mortality of target diseases. For every case of poliomyelitis reported, a detailed epidemiological investigation is done by the medical officer. The "polio-plus" committee situated at headquarters carries out a regular review of the cases for surgery and calipers. ${ }^{10}$

\section{IMMUNISATION AND LAMENESS SURVEYS}

The immunisation coverage surveys were carried out in the four regions of the Union Territory of Pondicherry for the five years from 1988-92. Lameness surveys were conducted for three successive years from 1989-91 during January and February of each year.

A list of villages and urban wards was compiled along with the population figures from the census report of 1981 which formed the sampling frame. ${ }^{11}$ Fresh samples of 30 clusters were selected each time the survey was done as per the method suggested in the Government of India module. ${ }^{12}$ These clusters were allocated to different regions of the Union Territory based on the size of the population Pondicherry main had 22 clusters, Karaikal 5, Mahe 2, and Yanam 1.

Children aged 12 to 23 months were included in the assessment of immunisation. The ages of the reference children included in the survey were verified by their birth certificates or health centre and anganwadi records. The

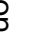


parents of the children were asked to show the immunisation card. If the card was not available the surveyor asked the parents (mostly mothers) about the immunisation details. Information was verified from records available at the health centre and anganwadi. Survey tools included a coverage survey household form, one for immunisation and another for lameness survey, a list of lame children, and clinical observation of lame children. All of these schedules were prepared on the lines given in the UIP manuals of the Government of India, based on WHO modules. ${ }^{12}$

For the immunisation coverage survey, seven eligible children and their mothers were included from each cluster. The first child was identified by random method, the remainder by systematic sampling including the first six children and mothers living in the next consecutive households. For lameness survey, about 335-340 children in each cluster below the age of 60 months were selected. If a sufficient number of children was not available in one village, contiguous village(s) were included.

The criteria for poliomyelitis employed in this study were:

- History of abrupt febrile illness;

- History of abrupt onset of weakness or paralysis of the leg(s) and/or arms;

- No progression of paralysis;

- Paralysis not associated with trauma; and

- Paralysis not present from birth or associated with mental retardation.

The survey was conducted by six to eight teams consisting of faculty members from the Departments of Preventive and Social Medicine and Paediatrics, postgraduate students of Preventive and Social Medicine and Paediatrics, medical interns, staff of the Jawaharlal Institute. The anganwadi workers of ICDS programme also accompanied the teams to facilitate the identification of the study population. Before the survey, a briefing session was held to familiarise members with the survey technique. Each case of lameness identified by the survey teams was examined by a faculty member from the respective teams, who also filled up a separate form containing information on clinical observations. Causes other than poliomyelitis were eliminated.

\section{ANALYSIS}

La Force has suggested the following methods for calculating the prevalence of all paralytic cases due to poliomyelitis and the incidence of poliomyelitis. ${ }^{13}$ Survey of residual paralysis due to poliomyelitis is reported as cases per 1000, usually in children older than 5 years. This prevalence rate represents the sum of all cases, almost all of which occurred from 0-4 years of age. The prevalence rate of residual paralysis can be used to estimate the annual incidence of paralytic cases. For example, if a prevalence rate of 10 cases per 1000 is found in a cohort of children 5-10 years of age, correction for those cases not involving the lower extremities is done by multiplying the prevalence rate by 1.25 per 1000 . An estimate of the annual in-
Table 1 Details of lameness surveys in Pondicherry, 1989-91

\begin{tabular}{lrrr}
\hline & \multicolumn{3}{l}{ Survey year } \\
\cline { 2 - 5 } & 1989 & 1990 & \multicolumn{1}{l}{1991} \\
\hline Households & 11746 & 13604 & 18637 \\
Children aged <60 mths & 11461 & 10093 & 11218 \\
Lameness due to poliomyelitis & 50 & 24 & 17 \\
Prevalence of poliomyelitis & 4.4 & 2.4 & 1.5 \\
Prevalence of polio cases & 5.9 & 3.2 & 2.0 \\
Males \% & 56.0 & 54.2 & 41.2
\end{tabular}

cidence could be obtained by dividing the prevalence rate ( 12.5 per 1000$)$ by the number of years at risk (five), giving an annual incidence of 2.5 per 1000 in the $0-4$ year age group. If the $0-4$ year old population makes up $20 \%$ of the population, the annual incidence is $2.5 /$ $1000 \times 0.2=0.5$ per 1000 population or 50 per 100000 population. An estimate of all cases of paralytic poliomyelitis can be made by multiplying annual incidence by 1.33 to correct for those cases that recovered completely or died within two months after onset of disease.

Since the present study included children in the $0-60$ months age group and definition of a case included paralysis of the upper or lower limb, certain modifications were made in the criteria suggested by La Force. The number of paralytic cases was not multiplied by a factor of 1.25. Prevalence was calculated by using multiplying factor of 1.33 . The incidence was not calculated as all the children in the survey had not reached 60 months of age.

\section{Results}

Table 1 shows the number of the households visited, the number of children below 60 months of age who were examined by the visiting teams, the number of children detected who were lame as a result of poliomyelitis, prevalence of poliomyelitis and gender of cases.

\section{NUMBERS SURVEYED}

More than 10000 children took part in each survey. The prevalence of lame children was 4.4 per 1000 children in 1989 . This had reduced progressively to 2.4 in 1990 and 1.5 in 1991 . Compared with 1989, the number of lame children fell by $55.6 \%$ in 1990 and $66 \%$ in 1991 Lame children were equally distributed in both the sexes. The male:female ratio was 1.1:1.

\section{AGE DISTRIBUTIONS}

Table 2 shows the distribution of paralytic cases according to the then age. There was not a

Table 2 Age distribution of paralytic polio cases

\begin{tabular}{lcccccc}
\hline Age (mth) & \multicolumn{3}{l}{ Survey year } & & \multicolumn{2}{c}{ Total } \\
\cline { 2 - 4 } \cline { 7 - 8 } & 1989 & 1990 & 1991 & & No & $(\%)$ \\
\hline $0-12$ & 0 & 0 & 0 & & $(0.0)$ \\
$13-24$ & 8 & 1 & 0 & 9 & $(10.1)$ \\
$25-36$ & 16 & 2 & 1 & 19 & $(20.8)$ \\
$37-48$ & 9 & 7 & 6 & & 22 & $(24.1)$ \\
$49-60$ & 17 & 14 & 10 & & 41 & $(45.0)$ \\
\hline
\end{tabular}


Table 3 Age at onset of poliomyelitis*

\begin{tabular}{|c|c|c|c|c|c|}
\hline \multirow[t]{2}{*}{ Age (mth) } & \multirow[t]{2}{*}{ No of $c a$} & \multicolumn{4}{|c|}{ Cumulative total } \\
\hline & & \multicolumn{2}{|c|}{ No } & \multicolumn{2}{|c|}{ (\%) } \\
\hline $\begin{array}{l}3 \\
6 \\
9 \\
12 \\
15 \\
18 \\
36 \\
60\end{array}$ & $\begin{array}{r}3 \\
11 \\
18 \\
22 \\
24 \\
17 \\
12 \\
2\end{array}$ & \multicolumn{2}{|c|}{$\begin{array}{r}3 \\
14 \\
32 \\
54 \\
57 \\
75 \\
87 \\
89\end{array}$} & \multicolumn{2}{|c|}{$\begin{array}{r}(3.4) \\
(15.7) \\
(36.0) \\
(60.7) \\
(65.2) \\
(84.3) \\
(97.8) \\
(100.0)\end{array}$} \\
\hline \multicolumn{6}{|c|}{$\begin{array}{l}\text { Median age at onset }=10.7 \text { months } \\
{ }^{*} \text { Information of two cases was not available. }\end{array}$} \\
\hline \multicolumn{6}{|c|}{$\begin{array}{l}\text { Table } 4 \text { Oral poliomyelitis vaccine status before the onse } \\
\text { of lameness }\end{array}$} \\
\hline \multirow[t]{2}{*}{ Vaccination } & & \multicolumn{2}{|l|}{ Year } & \multicolumn{2}{|c|}{ Lame children } \\
\hline & & 1990 & 1991 & No & $(\%)$ \\
\hline \multicolumn{2}{|c|}{$\begin{array}{l}\text { Received } 3 \text { doses } \\
\text { Received } 1 \text { or } 2 \text { doses } \\
\text { Total }\end{array}$} & $\begin{array}{l}14 \\
10 \\
24\end{array}$ & $\begin{array}{r}8 \\
9 \\
17\end{array}$ & $\begin{array}{l}22 \\
19 \\
41\end{array}$ & $\begin{array}{r}(54.4) \\
(45.6) \\
(100.0)\end{array}$ \\
\hline
\end{tabular}

single case among infants in the three surveys. Among children aged 13-24 months, there was only one case in 1990 and no case in 1991, whereas there were eight cases in this age group in 1989. In children up to the age of 36 months, there were 24 cases in 1989 compared with three cases in 1990 and one in 1991. These differences between 1989 and 1990 and 1989 and 1991 were statistically significant. (Value of $\mathrm{Z}$ for comparison between 1989 and $90=$ $3.61 \mathrm{p}<0.001 ; Z$ value for comparison between 1989 and $1991=4.72, \mathrm{p}<0.001$.)

The age at onset of cases is given in table 3 . About $16 \%$ of cases developed lameness before the age of 6 months; $61 \%$ by 12 months, $84 \%$ by 18 months, and $97.8 \%$ by 36 months. The median age at onset was 10.7 months.

\section{VACCINATION COVERAGE}

Vaccination status before the onset of lameness is shown in table 4. This information was available for the surveys conducted in 1990 and 1991 only. About $54 \%$ of lame children had received three doses of OPV before paralysis occurred and others had received either one or two doses.

Table 5 shows the immunisation coverage of infants with OPV and the drop-out rates. A total of 210 children aged 12-23 months was included each year, except in 1992 when 208 children were covered. The reason for ex-

Table 5 Details of infant's immunisation coverage and the drop-out rates of oral poliomyelitis vaccination (OPV)

\begin{tabular}{|c|c|c|c|c|c|}
\hline & \multicolumn{5}{|l|}{ Year } \\
\hline & 1988 & 1989 & 1990 & 1991 & 1992 \\
\hline Households surveyed & 1381 & 1682 & 1916 & 1861 & 2501 \\
\hline Eligible children surveyed & 211 & 210 & 210 & 210 & 208 \\
\hline Families with an immunisation card (\%) & 55 & 70 & 73 & 81 & 82 \\
\hline \multicolumn{6}{|l|}{ Immunisation (polio) diose: } \\
\hline 1st & 97.0 & 100.0 & 99.5 & 99.0 & 99.5 \\
\hline 2nd & 95.0 & 99.0 & 99.5 & 99.0 & 99.0 \\
\hline 3 rd & 91.0 & 97.0 & 98.0 & 98.0 & 97.6 \\
\hline \multicolumn{6}{|l|}{ Drop-out rates for OPV: } \\
\hline 1st-2nd & 2.4 & 1.0 & 0.5 & 0.0 & 0.5 \\
\hline 2nd-3rd & 4.0 & 2.4 & 1.0 & 1.0 & 1.4 \\
\hline $1 \mathrm{st}-3 \mathrm{rd}$ & 6.3 & 3.4 & 1.4 & 1.0 & 1.9 \\
\hline
\end{tabular}

cluding two children in 1992 was that they were not in the prescribed age limit. The percentage of families who had an immunisation card rose from 55 in 1988 to 82 in 1992. The percentage of children who received the first dose of OPV was very high, with only small variations (ie $97 \%-100 \%$ ); that for the third dose ranged from $91 \%-98 \%$. Drop-out rates for OPV from the first to the third dose were about $6-7 \%$ during 1988 , about $3.5 \%$ in 1989 , and about $1-2 \%$ in subsequent years. Dropout rates for OPV from the first to the second doses and the second to the third doses, which were 2.45 and $4 \%$ respectively in 1988 , reduced further to $0.5 \%$ and $1.4 \%$ respectively in 1992 .

\section{Discussion}

$\mathrm{John}^{14}$ reviewed the poliomyelitis situation in India up to 1991 . The reported numbers of cases of poliomyelitis were about 20000 in 1979 and 38000 in 1981. They gradually declined to the pre-immunisation level of 20000 in 1986 , but increased to 28000 in 1987 . In 1989 , the reported number of cases fell below the pre-immunisation level, and the number has further declined to 5669 in 1991. The three dose, OPV coverage during infancy was estimated to be about $80 \%$ in $1991 .^{14}$

The prevalence of lameness of 4.4 per 1000 children in 1989 that we found is comparable to $3.9 / 1000$ children aged under 6 years reported in a community survey in Pondicherry conducted by Balasoudarssanane,${ }^{4}$ and to the figure of 3.8 per 1000 children in the $0-4$ years group in the adjoining district of South Arcot, Tamil Nadu. ${ }^{2}$ In 1991 , however, it was 1.5 per 1000 children, which is a reduction of $66 \%$ compared with the 1989 level.

The UIP surveillance system in Pondicherry has also reported a gradual reduction in the number of new poliomyelitis cases between 1986-87 and 1989-90. The numbers of new cases reported were $39,74,37$, and 19 in 1986-87, 1987-88, 1988-89, and 1989-90 respectively. ${ }^{10}$ In the neighbouring state of Tamil $\mathrm{Nadu}$, there was a gradual reduction in the incidence of cases in the total population in 1987-92. The incidence in 1987 was 8.2 per 100000 population and in 1992 it was 0.8 . Most of the cases $(78 \%)$ in 1992 were reported from Chengalput, Madras, South Arcot, Thiruvannamalai, and Vellore districts. ${ }^{15}$

The reduction in prevalence seen in the present study can be attributed to high levels of vaccine coverage of about $91-98 \%$ among infants between 1988 and 1991. This coverage is the highest, compared with $47 \%$ in UIP districts and $27 \%$ in non-UIP districts in 11 major states of India. ${ }^{16}$ But the distribution of vaccine coverage had improved by 1989 to less than $50 \%$ in $19 \%$ of the districts, more than $50 \%$ in $56 \%$ of districts, and more than $85 \%$ in $25 \%$ of the districts. ${ }^{2}$ Similarly, the drop-out rates for OPV were far lower in this study compared with those of the other studies conducted in 1988 and 1989. In the present study, the drop-out rates between the first and the third dose of OPV were $6-7 \%$ in $1988,3-4 \%$ in 1989 , and $1-2 \%$ in the subsequent three 
years. In the ICMR study, however, the dropout rates between the first and second doses of OPV were 11 to $18 \%$ and double this between the second and third doses. ${ }^{16}$ In the national survey the drop-out rates between the first and third doses varied from $11-30 \%$.

The national survey in 1989 has shown that there was an inverse relationship between the prevalence of lameness and level of immunisation with 3 doses of OPV. In districts where the levels of immunisation were between $30 \%$ and $50 \%$, the prevalence was highest (7-9 per 1000 children), and at the coverage level of $80-90 \%$, the prevalence was $0.2-0.6$ per 1000 children. $^{2}$ A community based survey carried out in children aged 5-9 years showed an incidence of 0.56 per 1000 aged $0-4$ years where the immunisation level was $70 \% .{ }^{17}$ In a rural community block of Haryana the prevalence of lameness was 7.3 per 1000 , children born in 1974-76, 7.7 per 1000 children born in $1977-80$, and 2.3 per 1000 children born in 1981-84. Immunisation coverage with three doses of OPV was less than $10 \%$ during 1974-80 when immunisation was a clinicbased activity. Coverage increased from 5080\% during 1981-85 when OPV was given during an annual immunisation campaign. ${ }^{18}$ Another evaluation done in 1989 in the same area revealed that the prevalence was further reduced to 1.4 per 1000 born during 1984-88 when the level of vaccination coverage with three doses of OPV was 77-89\%. ${ }^{19}$ In Bombay, the immunisation coverage with three doses of OPV in children below 2 years of age had reached $80 \%$ by the year 1989 . As a result, the high incidence of over 11 per 100000 population prevailing in the six year period 1982-87 has declined to around 6 per 100000 in 1988 and 1989. The average age specific incidence (for the age group below 1 year) was found to be 1.04 per 1000 children in 1988-89 compared with $2.04 \%$ in the preceding six years. $^{20}$

There was a change in the distribution of lame children in relation to age in 1990 and 1991. A considerable decline in the number of affected children up to the age of 36 months was seen. It was $12.5 \%$ in 1990 and $6 \%$ in 1991 compared with $48 \%$ in 1989 . These results may indicate a remarkable reduction in the transmission of the disease. The age at onset was less than 1 year in most $(60.7 \%)$. The median age at onset was 10.7 months. Mahadevan reported that the median age of onset was 11 months for the period $1978-87,{ }^{3}$ and Soudarssanane reported a value of 13.4 months. ${ }^{4}$

There was no difference in the distribution of lame children in relation to sex. Earlier studies in Pondicherry have reported a preponderance of boys - the male:female ratio being 1.4-1.5:1. ${ }^{3421}$

This study showed that about $54 \%$ of lame children were given three doses of OPV before the onset of paralysis. Mahadevan et al reported that $14.7 \%$ cases of poliomyelitis had received three doses of OPV, ${ }^{3}$ and Soudarssanane et al reported that $41 \%$ had received three doses of OPV. ${ }^{4}$ Prevalences were $14 \%$ in 1988 in a series of cases admitted to a hospital in New Delhi ${ }^{6}$ and $26 \%$ in a series of cases admitted to a hospital in Madras during $1988-89 .^{22}$ It is argued that this may not be due entirely to vaccine failure, but there may be other risk factors such as intramuscular injection. Mahadevan et al, Soudarssanane et al , and Deivanayagam et al reported that intramuscular injection might have provoked poliomyelitis in 66,54 , and $70 \%$ of cases respectively. ${ }^{3422}$

Using the data of the poliomyelitis surveillance in Bombay for the period 1982-87, the estimated efficacy of three doses of OPV in children aged $12-23$ months exceeded $90 \%$. The authors reported that in Bombay, poliomyelitis results primarily from a failure to vaccinate fully all eligible children. ${ }^{23}$ In a casecontrol study conducted in Vellore (South India) in 1988, the vaccine efficacy for three doses of OPV was $62 \%$ for all under $5 \mathrm{~s}$, and for the $12-23$ months age group it was $71.4 \% .^{24}$ The authors suggested a five dose OPV schedule and annual OPV immunisation campaigns in addition to the routine three dose schedule.

The prevalence of poliomyelitis fell to 2.0 per 1000 in the beginning of 1991. Further reduction appears possible if the present level of immunisation is continued or if it is raised to $100 \%$. Apart from routine immunisation, zero dose at birth and immunisation for inaccessible children during certain months of the year, introduced in Pondicherry in 1990, will help in achieving $100 \%$ coverage and thereby decreasing the prevalence still further. Pulse polio immunisation carried out throughout the country in December 1995 and January 1996 and its continuation for some more years has the greatest potential in eradicating wild polio virus and thereby achieving zero polio by the year 2000 . There is scope for further improvement in the surveillance system. Rehabilitation of the affected children by provision of crutches or calipers and surgery needs to be increased. This will not only help these children but will increase the awareness of the community. It may also increase community participation in controlling the disease.

In conclusion, lameness surveys conducted at yearly intervals helped in assessing the poliomyelitis situation in the community. There was a rapidly decreasing trend from 1989-91. Children up to the age of 36 months formed the majority of the affected children. Further investigations are necessary to explain the causes of vaccine failure. This reduction may be attributed to efficient implementation of universal immunisation programme in the region.

We thank the staff of the Departments of Preventive and Social Medicine and Pediatrics for their active participation in the surveys; the Director of Social Welfare, the staff of ICDS cell; Deputy Director of Immunisation Govt of Pondicherry for the logistic support and the Anganwadi workers for their kind help and cooperation. We also thank the Director of JIPMER, Pondicherry, Dr D S Dubey, for his encouragement. Funding: this work was carried out as part of the universal immunisation programme funded by part of the universal and Family Welfare, Government of India and UNICEF. Conflicts of interest: none.

1 Robertson SE, Chan C, Kim-Farley R, Ward N. Worldwide status of poliomyelitis in 1986, 1987 and 1988 and plan for its global eradication by the year 2000 . World Health Stat $Q$ 1990;43:80-90. 
2 Gupta JP, Indira M. National review of immunisation programme in India. New Delhi: National Institute of Health gramme in India. New Dell

3 Mahadevan S, Shanthi A, Srinivasan S. Poliomyelitis: 20 years-the Pondicherry experience. $\mathcal{f}$ Trop Med Hyg 1989; 92:416-21.

4 Soudarssanane MB, Rotti SB, Srinivasa DK, Ramalingam G. Paralytic poliomyelitis in children under 6 years in Pondicherry. A community survey. $\mathcal{f}$ Epidemiol Community Health 1993;58:609-20.

5 Sulekha C, Sujamol S, Sugunabai NS, Cherian T, John TJ. An epidemic of poliomyelitis in Southern Kerala. Int $\mathcal{J}$ An epidemic of poliomyel
Epidemiol 1990;19:177-81.

6 Sharma M, Sen S, Ahuja B, Dhamia K. Paralytic Poliomyelitis 1976-1988; report from sentinel centre. Indian Pediatr 1990;27:143-50.

7 Government of Pondicherry. Annual Report 1992-93. Pondicherry: Directorate of Health and Family Welfare Services, 1993.

8 Soudarssanane MB, Srinivas DK, Narayan KA, Ramalingam G. Infant mortality in Pondicherry. An analysis of ingam G. Infant mortality in Pondicherry. An analysis of

9 Sachadev Y, Tandon BN, Krishnamurthy KS, Kapil V. Monitoring, motivation, continuing education, evaluation, research and training system in ICDS. New Delhi: Central Technical Committee, ICDS, Department of Women and Child Development, Ministry of Human Resource Development, Government of India, 1992.

10 Government of Pondicherry. National mission on immunisation 1989-90. Pondicherry: Directorate of Health munisation 1989-90. Pondicherry:
and Family Welfare Services, 1990

11 Government of Pondicherry. Statistical handbook 1982-83, Pondichery: Directorate of Economics and Statistics, 1985.

12 Government of India. Evaluate vaccine coverage. New Delhi: Ministry of Health and Family Welfare, 1989.
13 La Force FM, Lichnevski MS, Kaje J, Henderson RH. Clinical Survey Technique to estimate prevalence and annual incidence of poliomyelitis in developing countries. Bull World Health Organ 1980;58:609-20.

14 John TJ. Experience with polio vaccine in the control of poliomyelitis in India. Public Health Rev 1993-94;21:8390.

15 Anonymous. Acute faccid paralysis in children. CSSM Review 1993;5;1-7.

16 Patowari AC, Jaiswal ON, Sundar Lal, et al. Coverag evaluation survey of immunisation in eleven states of India. Indian fournal of Community Medicine 1990;15:222-26.

17 Acharya D, Chakladdar BK. An epidemiological study of paralytic poliomyelitis cases in Kasturba Hospital, Manipal. $\mathcal{F}$ Commun Dis 1989;21:183-89.

18 Khajuria R, Datta N, Kumar R, Kaur T, Kaushal MK, Singhi S, Kumar V. Impact of annual immunisation programme with oral polio vaccine on the prevalence of paralytic poliomelitis. Indian 7 Pediatr 1989;56:342-47.

19 Kumar R, Kumar V. Poliomyelitis control by annual immunisation campaign with oral polio-virus vaccine in a rural area of India. Trop Geogr Med 1991;43:215-19.

20 Mandke BV, Pawar RM, Naik DD, Torne VP, Naiksatam AM. Review of poliomyelitis in Bombay (1982-89): ObAM. Revion the improved immunisation coverage. Indian servations on the improved immunisation

21 Rotti SB, Satpathy SK, Mehta SP. Prevalence of paralytic poliomyelitis in Pondicherry, South India. $f$ Epidemiol poliomyelitis in Pondicherry, Sou

22 Deivanayagam N, Nedunchelian K. Epidemiological and clinical features of acute poliomyelitis in children admitted in a urban hospital. Indian Pediatr 1992;29:25-28.

23 Kim-Farley RJ, Dave KH, Sokhey J, Mandke VB. Polio1982-87. Bull World Health Organ. 1989;67:663-67.

24 Balraj V, John TJ, Thomas M, Mukundan S. Efficacy of oral polio virus vaccine in rural communities of North Arcot District, India. Int $\mathcal{F}$ Epidemiol 1990;19:7111-4. 